\title{
BENEFíCIOS DA FASEOLAMINA (Phaseolus vulgaris L.) - UMA REVISÃO
}

\section{BENEFITS OF PHASEOLAMINE (Phaseolus vulgaris L.) - REVIEW STUDY}

\author{
COLAÇO, Priscila Caon'; DEGÁSPARI, Cláudia Helena²
}

1-Discente do Curso de Farmácia das Faculdades Integradas do Brasil - UniBrasil e-mail: priscila.caoncolaco@hotmail.com

2-Professora Colaboradora das Faculdades Integradas do Brasil - UniBrasil

e-mail: sensus@cwb.matrix.com.br

\section{RESUMO:}

Este trabalho relata sobre os benefícios da faseolamina, proteína extraída do feijão branco (Phaseolus vulgaris L.), grão este considerado um importante componente da dieta alimentar do brasileiro. A faseolamina possui efeito na inibição da digestão e também na absorção de amido, sendo, portanto uma inibidora da enzima alfa-amilase. Ela tem sido utilizada como tratamento para indivíduos na perda de peso, bem como um efeito hipoglicemiante em diabéticos. A faseolamina é comercializada no Brasil na forma de cápsulas, que só podem ser manipuladas em farmácias magistrais, e a sua matéria-prima deve obtida de fornecedores que possuam laudo e certificado de análise que garantam sua qualidade. Em se tratando da posologia da faseolamina, os médicos prescrevem cápsulas com $500 \mathrm{mg}$, administradas duas vezes ao dia. A recomendação para ingerir a faseolamina deve ser 30 minutos antes das duas principais refeições do dia. Nesta revisão de literatura foram considerados artigos científicos principalmente dos últimos cinco anos, com a finalidade de expandir a percepção dos benefícios e a aplicabilidade da faseolamina. A ingestão frequente desta proteína (através do consumo do feijão) impacta diretamente na qualidade de vida do brasileiro, pois o visível decréscimo no consumo de feijão é, indiretamente, uma das causas do aumento significativo da obesidade observado na população nos últimos anos.

Palavas-chave: Phaseolus vulgaris L., feijão branco, faseolamina, alfa-amilase .

\section{ABSTRACT:}

Bean is considered an important component of the Brazilian diet, and presents in a protein named phaseolamine, one inhibitory of alpha amylase. This protein is extracted from bean, and has inhibitory effects in the digestion and absorption of starch. Has been used in treatment for people in weight loss and shows hypoglycemic effects in diabetics. The phaseolamine is marketed in Brazil like capsules and can only be manipulated in special pharmacies, and the raw material suppliers do need to have report and certificate of quality securities analysis. For the faseolamine medicine dosage, the doctors prescribe capsules with 500 milligrams, administered twice a day. The recommendation is taking with water 30 minutes before the two main daily meals. This review study was written based in the scientific articles mainly from the last five years, with the purpose of expanding the perception of the benefits and the applicability of the faseolamine. The frequently ingestion of this protein (through beans consumption), results directly in a life quality increase in the Brazilian people, because the visible decrease in the bean consumption is, probably, one of the significant causes of the increase of the obesity in the population in the last years.

Keywords: Phaseolus vulgaris L., white bean, phaseolamine, alpha-amylase. 


\section{INTRODUÇÃO}

Atualmente a faseolamina está sendo prescrita por médicos, como coadjuvante nas dietas com objetivo de redução de peso e também para pacientes com diabetes. Encontram-se no mercado hoje, diversos produtos denominados como suplementos alimentares para emagrecimento, entre os quais tem destaque principalmente para os que possuem compostos à base de extratos vegetais. Cabe salientar que a faseolamina deve ser usada como auxílio no tratamento da obesidade $e$ não como estrutura principal (ARBO et al, 2009; PEREIRA, 2008).

A proteína faseolamina é dita como inibidora da enzima alfa-amilase. Essa enzima tem destaque no metabolismo de carboidratos em animais, em plantas e em outros organismos. Suplementos contendo inibidores de alfa-amilase foram comercializados como "bloqueadores de amido", sendo utilizado para o controle do Diabetes e também da obesidade (OCTÁVIO, 2002; LIENER; DONATUCCI \& TARCZA, 1984; QIAN, 2013).

Tanto em países desenvolvidos, quanto em países em desenvolvimento, é possível observar em estudos epidemiológicos, um aumento de peso da população. Contudo a faseolamina se faz importante, no tratamento da perda de peso, melhorando o padrão alimentar de indivíduos, e também para o controle do Diabetes (BLUNDELL \& GILLET, 2001; NONINO-BORGES; BORGES \& SANTOS, 2006).

Portanto este trabalho tem como objetivo fazer uma revisão de literatura sobre os benefícios da faseolamina na redução de peso, citando seus efeitos no controle de glicemia, e descrever os resultados de estudos, que foram publicados principalmente nos últimos anos citando esta proteína do feijão branco (Phaseolus vulgaris L.).

\section{REVISÃO DE LITERATURA}

Atualmente há uma busca quase que frenética pelo corpo perfeito, principalmente estimulado pela mídia, onde, a magreza está inserida na sociedade como padrão e parâmetro de beleza. Com isso, existe uma busca e consumo muito grandes e desenfreados de alternativas, que sejam práticas e que tenham fórmulas ditas "milagrosas", deve-se ter certa cautela e atenção profissional para com estes produtos. A maioria está baseada somente em propagandas enganosas, onde não se apresenta comprovação da eficácia farmacológica e segurança toxicológica (PELIZZA, 2010).

Diversos estudos relatam uma variedade de produtos e extratos de origem natural, incluindo compostos isolados de plantas, que estão sendo utilizados no tratamento da perda de peso, assim como prevenindo a obesidade gerada por uma dieta rica em calorias (HAN, 2013). 
Desde 1945, os inibidores de alfa-amilases começaram a ser mencionados na literatura científica. Segundo dados de BOWMAN, constatou-se a presença de um inibidor hidrossolúvel e termolábil nos feijões (BOWMAN, 1954).

Em trabalho com o feijão branco, MARSHAL \& LAUDA (1975), descreveram a purificação, bem como algumas propriedades físico-químicas do inibidor de alfaamilase, sendo denominado faseolamina. Entretanto, observou-se que a faseolamina possui alta especificidade pelas alfa-amilases do pâncreas de porcos, de seres humanos e também de saliva humana (MARSHALL \& LAUDA, 1975).

Suplementos contendo inibidores de alfa-amilase, por volta da década de 1980, foram comercializados como "bloqueadores de amido", sendo utilizado para o controle do Diabetes e também da obesidade. A grande maioria desses produtos consistia extratos de feijão com uma baixa atividade antiamilásica, e com uma grande quantidade de lectina e inibidores de tripsina, sendo considerados danosos (LIENER et al, 1984).

Segundo MARSHAL \& LAUDA (1975) e PUSZTAI (1995) os processos de purificação do inibidor de alfa-amilase, descritos por eles, envolveram centrifugação, cromatografia de filtração molecular, cromatografia de troca iônica, diálise e liofilização. Com estas especificações foi possível sugerir um estudo comparativo entre a faseolamina comercial e as farinhas de feijões crus (PEREIRA, 2008; MARSHALL \& LAUDA, 1975).

LAYER, CARLSON \& DIMAGNO (1985), por meio de uma perfusão de inibidor de alfa-amilase parcialmente purificado no duodeno, mostrou em estudos em seres humanos, uma inibição significativa da atividade da amilase, durante a digestão de amido (LAYER; CARLSON \& DIMAGNO, 1985).

Em outro trabalho, LAYER, CARLSON \& DIMAGNO (1985) descrevem que, com a ingestão de $50 \mathrm{~g}$ de amido, juntamente com o inibidor purificado, houve uma redução dos níveis de glicemia pós prandial, em indivíduos com Diabetes (LAYER; ZINSMEISTER \& DIMAGNO, 1986).

Em se tratando das fibras presentes nos grãos de feijão, LAJOLO, GENOVESE \& MENEZES (1996) destacaram em seu presente trabalho, o reconhecido efeito hipercolesterolêmico e hipoglicêmico, e também da presença de vitaminas do complexo B, e um alto conteúdo de carboidratos complexos (LAJOLO; GENOVESE \& MENEZES, 1996).

A proteína faseolamina é dita como inibidora da enzima alfa-amilase. OCTÁVIO et al (2002), mostra que as alfa-amilases são enzimas monoméricas que fazem parte de uma família de endoamilases que a hidrólise de ligações glicosídicas $\alpha$ 1,4 dos carboidratos. Essas enzimas têm destaque no metabolismo de carboidratos em animais, em plantas e em outros organismos. As alfa-amilases estão presentes na saliva e também na secreção pancreática de mamíferos. A alfa-amilase está presente 
também em vários tecidos como: pulmão, cérebro e glândulas salivares, contudo encontra-se em maior quantidade no pâncreas e no duodeno (OCTÁVIO, 2002; QIAN, 2013; BROBST et al, 1997).

Udani e Singh (2007) realizaram um estudo com 25 indivíduos saudáveis, divididos em dois grupos, sendo o primeiro denominado grupo placebo, e o outro grupo experimental. Estes foram administrados $500 \mathrm{mg}$ de extrato de feijão branco, duas vezes ao dia, em conjunto com um programa de emagrecimento, onde incluía: dieta, exercício físico e intervenção comportamental. Pode-se concluir nesse estudo, que houve perda de peso e redução da circunferência de cintura nos dois grupos. Porém a maior redução foi observada no grupo experimental, o qual consumia mais quantidade de carboidrato.

Em indivíduos com sobrepeso, houve uma redução significativa do peso corporal e também nos níveis séricos de triglicerídeos no grupo experimental, em relação ao grupo placebo, onde não houve redução (UNDANI; HARDY \& MADSEN, 2004).

Segundo CELLENO et al (2007), em um estudo envolvendo 60 participantes com sobrepeso, que receberam uma dieta hiperglicídica, controlados com placebo durante 30 dias, verificou-se uma redução no IMC, tecido adiposo. Por outro lado, o grupo dos participantes que receberam o placebo, a massa muscular foi mantida (CELLENO et al, 2007).

Segundo um estudo realizado por PEREIRA (2008), camundongos foram classificados em grupos, sendo que cada grupo foi submetido aos seguintes tratamentos: grupo controle (que recebeu água destilada), grupo faseolamina (que recebeu extrato de faseolamina na proporção de $1 \mathrm{~g} / 70 \mathrm{~kg} / \mathrm{dia}$ ) e outro grupo que recebeu extrato de feijão branco $1 \mathrm{~g} / 70 / \mathrm{kg} / \mathrm{dia}$. Os extratos de feijão branco, faseolamina e água destilada, foram administrados via oral por gavagem, (que consiste na introdução de uma cânula metálica, com diâmetro de $3 \mathrm{~mm}$, conectada a uma seringa, por meio do esôfago do animal) recebendo uma vez ao dia (PEREIRA, 2008).

Esse experimento foi realizado em dias intercalados, efetuando-se o controle do peso corporal dos animais para posteriormente elaborar uma curva de crescimento. Foi aferido em balança de precisão, o peso corporal, onde a variação de peso foi utilizada para avaliar o desenvolvimento e o crescimento dos animais. Pode-se observar que nesse período a dose de um grama de farinha de feijão branco ou de faseolamina por $70 \mathrm{~kg}$ de peso corporal/dia não afetou o crescimento e não provocou efeitos prejudiciais aos camundongos tratados por 24 dias (PEREIRA, 2008).

Em uma pesquisa realizada por PAVANELLI; MEZA \& TIYO (2011) enfatiza-se que dentre os 252 fármacos essenciais selecionados pela Organização Mundial da Saúde, $11 \%$ são fitoterápicos. Em sua pesquisa, foi realizado um inquérito com 15 nutricionistas no município de Campo Mourão, Paraná, com o objetivo de se avaliar os 
fitoterápicos mais prescritos, onde a faseolamina foi citada (PAVANELLI; MEZA \& TIYO, 2011).

Assim, observa-se que a fitoterapia está se tornando atrativa ao tratamento da obesidade, por ser de caráter natural. Devido às formas diversas de comercialização destes produtos naturais atualmente, torna-se imprescindível à segurança, à qualidade e à garantia de eficácia, para que sejam utilizados de forma adequada, como coadjuvante na dieta para redução de peso de indivíduos (OLIVEIRA, 2013).

O Conselho Federal de Nutricionistas, através da Resolução CFN N402/2007 regulamenta a prescrição fitoterápica pelo nutricionista de plantas in natura frescas ou como droga vegetal nas suas diferentes formas farmacêuticas. No entanto, o Nutricionista não pode prescrever medicamentos alopáticos, mas pode criar fórmulas que contenham produtos fitoterápicos de acordo com a resolução descrita. Estas fórmulas devem ser um instrumento adjuvante no tratamento para redução de peso, sendo sempre associado de um plano alimentar equilibrado, como no caso da faseolamina (CONSELHO FEDERALDE NUTRICIONISTAS, 2013).

Encontram-se no mercado hoje, diversos produtos denominados como suplementos alimentares para emagrecimento, entre os quais tem destaque principalmente para os que possuem compostos à base de extratos vegetais. Uma das explicações para o crescimento do consumo desses produtos de origem vegetal é pelo fato das pessoas terem vontade de estabelecer uma forma mais saudável de vida, e ter o entendimento de que todo produto natural é considerado saudável e seguro. No entanto, as pessoas tem associada uma falsa ideia, de que tudo que é de origem natural, não prejudica a saúde, sendo que é necessária cautela para esta análise, pois mesmo sendo produtos naturais, não são livres de efeitos adversos (ARBO et al, 2009).

A Resolução da Diretoria Colegiada n48 de 2004 da Agência Nacional de Vigilância Sanitária (ANVISA) fornece a seguinte definição: "os fitoterápicos são todos os medicamentos preparados exclusivamente com plantas ou partes de plantas medicinais, como, por exemplo, cascas, raízes, sementes, frutos, (flores ou folhas) as quais tenham propriedades sendo reconhecidas como prevenção, diagnóstico, ou tratamento de doenças" (ANVISA, 2013).

Um dos efeitos adversos que a faseolamina pode apresentar é uma ligeira diarreia que pode ocorrer no primeiro dia de tratamento, e, mais especificamente, em indivíduos que apresentem uma dieta mais voltada para o consumo de amido e massas. Para indivíduos hipoglicêmicos e gestantes, a faseolamina não é recomendada. Em se tratando da posologia da faseolamina, os médicos prescrevem cápsulas com $500 \mathrm{mg}$, administradas duas vezes ao dia. Sendo recomendada sua ingestão com água, 30 minutos antes das duas principais refeições do dia (EMBRAFARMAPHARMACEUTICAL EXPERTISE, 2013).

Tanto em países desenvolvidos, quanto em países em desenvolvimento, é 
possível observar em estudos epidemiológicos, um aumento de peso da população. Estes estudos sobre a história alimentar populacional têm como objetivo determinar o padrão alimentar de cada indivíduo, e identificar as possíveis causas que estejam contribuindo para a obesidade dos mesmos (BLUNDELL \& GILLET, 2001).

Estudos descrevem que os produtos naturais, que apresentam efeitos ditos como "antiobesidade", podem ser colocados em cinco categorias, descritos em seus diferentes mecanismos; (1) aqueles que diminuem a absorção de lipídios, (2) os que apresentam diminuição na absorção de carboidratos, (3) os que aumentam o gasto energético, (4) aqueles que diminuem a diferenciação e proliferação de pré-adipócitos, (5) e os que diminuem a lipogênese e aumentam da lipólise (FINARDI, 1983).

É na área de ciência dos alimentos que esses inibidores têm sido mais estudados, pois são encontrados em vegetais, que são ingeridos como alimentos, e acabam, em contrapartida, inibindo enzimas do digestório $O$ inibidor de alfa-amilase de feijão é uma glicoproteína que possui peso molecular de 53.000 Dalton, possui pH do ponto isoelétrico em 4,35, sendo composto por 400 resíduos de aminoácidos, sem pontes dissulfeto na estrutura (OCTÁVIO, 2002).

As propriedades nutritivas relacionadas ao feijão comum estão baseadas no fato de serem fontes ricas em proteínas $(19,6 \%$ a $26 \%$ da matéria seca - MS), apresentando $64 \%$ a $71 \%$ da MS de carboidratos com baixo índice glicêmico e com baixo índice de gordura, de $1 \%$ a $2 \%$ da MS (VIEIRA, 1967).

As proteínas de Phaseolus vulgaris possuem baixo valor biológico, e isto é atribuído á vários fatores como: estrutura compacta de proteínas nativas de feijões, que podem resistir à proteólise, um baixo conteúdo de aminoácidos sulfurados, compostos antinutricionais que podem modificar a absorção de um nutriente e alterar a liberação dos aminoácidos (WU, 1995).

O coeficiente de absorção das proteínas do feijão, em ratos, está situado entre $40 \%$ e $70 \%$ (BRESSANI \& ELIAS, 1984).

A amilase salivar pancreática (APH) possui uma função importante, pois ela hidrolisa polissacarídeos grandes em oligossacarídeos menores, que em seguida são hidrolisados pelas a-glicosidades intestinais (NUMÃO et al, 2004).

Um outro estudo realizado num período de 20 dias com ratos diabéticos, tratados com faseolamina (100, 500 e $1500 \mathrm{mg} / \mathrm{kg}$ ) mostrou uma redução do índice glicêmico, acompanhada de uma redução do dano tecidual e de uma atenuação na formação de espécies reativas de oxigênio (EROs). O dano tecidual causado, que foi avaliado pela peroxidação lipídica teve um aumento nos ratos diabéticos que não receberam o tratamento com a faseolamina. Contudo, o dano no tecido foi reduzido nos ratos que receberam a faseolamina. Nesse estudo, foi possível observar um aumento significativo da deposição de colágeno em animais diabéticos, onde posteriormente houve uma redução da deposição de colágeno após o tratamento com a faseolamina 
nesses animais (OLIVEIRA, 2010).

Em um ensaio clínico, utilizando Phase $2{ }^{\mathrm{TM}}$, do fabricante Pharmachem Laboratories, foram selecionados 50 adultos obesos, para participar de um estudo randomizado, duplo-cego e placebo-controlado. Nesse ensaio, o objetivo foi controlado de forma a avaliar os efeitos do tratamento com o Phase $2 \AA \mathrm{TM}$ versus placebo na perda de peso. Os participantes receberam $1.500 \mathrm{mg}$ de Phase $2{ }^{\circledR} \mathrm{TM}$ e um placebo idêntico duas vezes ao dia. O período de estudo foi por 8 semanas, sendo que somente 27 indivíduos completaram o estudo. Os resultados após oito semanas demonstraram que o grupo que recebeu Phase $2 \circledR$ TM perdeu uma média de $£ 3,79$ (média de $£ 0,47$ por semana) em comparação com o grupo placebo, que perdeu uma média de $£ 1,65$ (média de $£ 0,21$ por semana), o que representa uma diferença de 129 por cento. Os níveis de triglicérides do grupo Phase $2 \AA^{\mathrm{TM}}$ foram reduzidos de uma média de $26,3 \mathrm{mg} /$ dl, uma redução três vezes maior do que os observados no grupo que recebeu o placebo (8,2 mg / dL). Pode-se concluir que Phase $2{ }^{\mathrm{T} M}$ contribuiu de forma eficaz para o tratamento da obesidade (JAY; MARY \& DAMIAN, 2004).

Uma pesquisa realizada pela Vigitel (Vigilância de Fatores de Risco e Proteção para Doenças Crônicas por Inquérito Telefônico) do Ministério da Saúde revelou que o percentual de pessoas com excesso de peso supera mais da metade da população brasileira. O estudo mostrou que $51 \%$ da população, acima de 18 anos, está acima do peso. Foram entrevistadas 45.400 pessoas em todas as capitais, entre julho de 2012 a fevereiro de 2013 (FINARDI, 1983).

O tratamento clínico é fundamental, dentre as opções terapêuticas para a obesidade, melhorando seu padrão alimentar, e também com estímulo de alguma atividade física. Contudo é preciso identificar quais os problemas encontrados no comportamento alimentar do paciente e também quais as possíveis outras falhas nos seus hábitos de vida. A obesidade é uma das enfermidades metabólicas mais antigas da humanidade, e as opções farmacológicas, para o seu tratamento são limitadas, e podem causar diversos efeitos colaterais indesejáveis aos indivíduos (NONINOBORGES; BORGES \& SANTOS, 2006).

O tratamento farmacológico deve servir como um auxílio ao paciente, no sentido de mudar sua dieta alimentar, e não reduzindo a fome. O paciente, tendo uma alimentação mais equilibrada, consequentemente adquiri mais bem estar e saúde a si próprio (NONINO-BORGES; BORGES \& SANTOS, 2006).

Outro dado relevante socioeconômico revela que o brasileiro está consumindo cada vez menos feijão. Segundo um levantamento da Companhia Nacional do Abastecimento (Conab), verificou-se uma queda da presença do feijão na dieta alimentar do Brasileiro (BEVILACQUA, ORILIO \& VERRE, 2010).

Importante observar que, segundo dados de outra pesquisa baseada no censo do Instituto Brasileiro de Geografia e Estatística (IBGE), entre 1972 a 2003, a redução 
no consumo do feijão foi em torno de $40 \%$, sendo que, especificamente no período de janeiro de 2009 a 2010, o consumo caiu em 5 \%. Esta questão pode, em parte, ser explicada, devido ao aumento de preço do feijão. Entre 2010 e 2013 a inflação que incidiu sobre os preços dos alimentos aumentou consideravelmente. Porém, isto não justifica a redução de seu consumo, uma vez que este aumento ocorreu também em diversos outros alimentos e nem por isso observou-se queda em seus consumos (BEVILACQUA, ORILIO \& VERRE, 2010).

Estudos apontam que esta redução do consumo de feijão pelos brasileiros pode estar associada ao aumento de cardiopatias, Diabetes e obesidade (DANTAS, 2013).

De acordo com profissionais ligados à área de saúde e nutrição, em cada 100 gramas de feijão, há 20 gramas de proteína, 60 gramas de carboidratos, 16 gramas de fibras, incluindo também vitaminas do complexo B (RUAS, 2013).

Com relação às comparações de valores nutricionais de alguns tipos de feijão, observa-se que a quantidade de proteína varia muito para cada tipo de grão. Por exemplo, o feijão preto apresenta 2,7 gramas de proteína, o carioca com 2,8 gramas, o popularmente denominado de rajado com 3,3 gramas, e o feijão branco que se destaca dentre os outros, com 14 gramas de proteína por cada 100 gramas (JORNAL HOJE, 2013).

\section{CONCLUSÃO}

A faseolamina, proteína extraída do feijão branco (Phaseolus vulgaris L.), mas presente nos outros tipos mostra-se importante e essencial na dieta alimentar do brasileiro, visto que, por todos os estudos realizados, exerce efeito na inibição da digestão do amido, bem como na sua absorção no digestório, por se tratar de uma proteína inibidora da enzima alfa-amilase.

Com o desenvolvimento de novas tecnologias, aplicadas principalmente à área alimentícia, os alimentos industrializados de rápido preparo e, na maioria das vezes, com um balanço nutricional não adequado, está conduzindo cada vez mais a mudanças drásticas no hábito de consumo dos brasileiros e, dentre estas mudanças encontra-se a redução do consumo do feijão, principalmente por se tratar de um alimento que requer um preparo mais demorado sendo, pouco a pouco, excluído das mesas e das cozinhas brasileiras. Com isso, indiretamente, deixa-se de consumir a proteína faseolamina (presente exclusivamente no feijão) e que, por este motivo, pode estar contribuindo para o aumento considerável da obesidade e todas as outras patologias oportunistas ligadas a ela (cardiopatias e Diabetes) entre os brasileiros.

Por tudo o que foi exposto, verifica-se o importante papel da faseolamina e recomenda-se que os brasileiros sejam cada vez mais alertados de que em sua dieta 
alimentar não pode deixar de conter feijão, pois esta leguminosa é fundamental para a melhoria de sua qualidade de vida e de uma alimentação equilibrada.

\section{REFERÊNCIAS}

ANVISA. Resolução da Diretoria Colegiada n48 de 2004, da Agência Nacional de Vigilância Sanitária. Disponível em: <http://portal.saude.gov.br/portal/arquivos/pdf/rdc_48 16_03_04_registro_fitoterapicos\%20.pdf>.Acessado em: abr. 2013.

ARBO, D.M. et al. Presence of p-synephrine in teas commercialized in Porto Alegre (RS/Brazil). Brazilian Journal of Pharmaceutical of Sciences, v.45, n.2, p. 273-278, 2009.

BEVILACQUA, C., ORILIO, C.; VERRE, C. Brasileiro consome cada vez menos feijão, mostra pesquisa. Piracicaba: Universidade Metodista de São Paulo, 2010. Disponível em: <http://www.metodista.br/rronline/noticias/economia/2010/06/brasileiro-consomecada-vez-menos-feijao-diz-pesquisa> Acessado em: set. 2013.

BLUNDELL, J.E.; GILLET, A. Control of food intake in the obese. Obes. n.9, p. 263-270, 2001.

BOWMAN, D.E. Amylase inhibitor in navy beans. Science, Washington. v. 102, p. 2649, 1945.

BRESSANI, R.; ELIAS, L.G. Relación entre La digestibilidad y el valor proteinico del frijol comum (Phaseolus vulgaris). Archivos Latinoamericanos de Nutrición, Venezuela. v.34, n.1, p. 189-197, 1984.

BROBST, D.F. et al. Pancreatic function.Clinical biochemistry of domestic animals. 5 ed. San Diego: Frontcover, p. 353-368, 1997. Disponível em: <>. Acessado em: jul. 2013.

CELLENO, L. et al. A dietary supplement containing standardized Phaseolus vulgaris extract influences body composition of overweight men and woman. Int J Med., v.4, p. 45-52, 2007. Disponível em: <http://www.ncbi.nlm.nih.gov/pmc/articles/PMC1796956/ pdf/ijmsv04p0045.pdf>. Acessado em: ago. 2013.

CONSELHO FEDERAL DE NUTRICIONISTAS. Resolução CFN n 402 de 2007. Disponível em: URL: <http://www.crn9.org.br/uploads/file/res402.pdf>. Acessado em: abr. 2013. 
DANTAS, T. Importância dos alimentos na saúde. Feijão. Disponível em: <http://www.mundoeducacao.com/saude-bem-estar/feijao.htm>. Acessado em: set 2013.

EMBRAFARMA PHARMACEUTICAL EXPERTISE. Faseolamina (Phaseolus vulgaris L. extract). Disponível em: <http://www.embrafarma.com.br/novo/modules/ pdf/d9d4f495e875a2e075a1a4a6e1b9770f.pdf>. Acessado em: set. 2013.

FINARDI, F.F. Parâmetros estruturais do inibidor de $\alpha$-amilase de feijão (Phaseolus vulgaris). [Dissertação] São Paulo: Universidade de São Paulo Faculdade de Ciências Farmacêuticas, 1983.

HAN et al. Anti-obesity effects of chikusetsusaponins isolated from Panax japonicus rhizomes. [periódico na internet]. BMC Complementary and Alternative Medicine, 2005; Disponível em: <http://www.biomedcentral.com/content/pdf/1472-6882-59.pdf>. Acessado em: set. 2013.

JAY, U.M.D.; MARY, H.M.D.; DAMIAN, C.B.A. Blocking Carbohydrate Absorption and Weight Loss: A Clinical Trial Using Phase $2^{\mathrm{TM}}$ Brand Proprietary Fractionated White Bean Extract. Alternative Medicine Review. v.9, n.1, p. 63-69, 2004. Disponível em: <http://www.ncbi.nlm.nih.gov/pubmed/15005645>. Acessado em: ago. 2013.

JORNAL HOJE. Consumo diário de arroz e feijão controla glicemia e previne doenças. 2011. Disponível em: <http://g1.globo.com/jornalhoje/noticia/2011/10/consumo-diario-de-arroz-e-feijao-controla-glicemia-e-previnedoencas.html>. Acessado em: set 2013.

LAJOLO, F.M.; GENOVESE, M.I.; MENEZES, E.W. Cultura do Feijoeiro Comum no Brasil. Piracicaba: Potafos. p. 71-99, 1996.

LAYER, P.; CARLSON, G.L.; DIMAGNO, E.P. Partially purified white bean amylase inhibitor reduces starch digestion in vitro and inactivates intraduodenal amylase in humans. Gastroenterology, v. 88, p. 1895-1902, 1985.

LAYER, P.; ZINSMEISTER, A.R.; DIMAGNO, E.P. Effects of decreasing intraluminal amylase activity on starch digestion and postprandial gastrointestinal function in humans. Gastroenterology, v. 91, p. 41-48, 1986.

LIENER, I.E. et al. Starch blockers: a potencial source of trypsin inhibitors and lectins. 
LIENER, I.E. et al. Starch blockers: a potencial source of trypsin inhibitors and lectins. American Journal of Clinical Nutrition, v.39, n.2, p. 196-200, 1984.

LIENER, I.E.; DONATUCCI, D.A.; TARCZA, J.C. Starch blockers:a potencial source of trypsin inhibitors and lectins. American Journal of Clinical Nutrition, v.39, n.2, p. 196200, 1984.

MARSHALL, J.J.; LAUDA, C.M. Purification and properties of phaseolamin, an inhibitor of a amylase, from the kidney bean (Phaseolus vulgaris). Journal of Biological Chemistry, Stanford, v.250, n.20, p. 8030-8037, 1975.

MINISTÉRIO DA SAÚDE. Mais da metade da população brasileira tem excesso de peso. Brasília, 2013. Disponível em: <http://portalsaude.saude.gov.br/portalsaude/ noticia/12926/162/mais-da-metade-da-populacao-brasileira-tem-excesso-depeso.html>. Acessado em: ago. 2013.

NONINO-BORGES, C.B.; BORGES, R.M.; SANTOS, J.E. Tratamento clínico da obesidade. Medicina, Ribeirão Preto, v.39, n.2, p. 246-252, 2006.

NUMÃO, S. et al. In situ extension as an approach for indefifying novel-amylase inhibitors. The Journal of Biological Chemistry. v.279, n.46, p. 48282-48291, 2004.

OCTÁVIO, L.F. et al. Plant $\alpha$-amylase inhibitors and their interaction with insect a-amylase. Structure, function and potential for crop protection. Eur. J. Biochem. v.269, p. 397-412, 2002.

OLIVEIRA, I.R. O uso de fitoterápicos como uma forma eficaz para o tratamento da obesidade: vantagens e desvantagens. Rev Eficaz. 2011. Disponível em: <http://www.faculdadeeficaz.com.br/revistacientificaeficaz/artigo/saude/2011/ed_03/l za-FINAL-2.pdf.>. Acessado em: set. 2013.

OLIVEIRA, R.J.S. Efeito da faseolamina sobre parâmetros bioquímicos gerais e oxidativos do coração de ratos diabéticos induzidos por STZ. [dissertação]. Uberlândia: Universidade Federal de Uberlândia, 2010.

PAVANELLI, F.M.; MEZA, L.K.S.; TIYO, R. Fitoterápicos mais prescritos por nutricionistas de um município paranaense, 2011. Disponível em: <http://www.cesumar.br/prppge/pesquisa/epcc2011/anais/sheila_karina_luders_mez a(1).pdf>. Acessado em: out. 2013. 
PELIZZA, M.C. Uso de Cereus sp e Cordia ecalyculata Vell. como emagrecedores: uma revisão. [tese livre docência]. Porto Alegre: Faculdade de Farmácia, Universidade Federal do Rio Grande do Sul, 2010.

PEREIRA, L.L.S. Estudo comparativo entre faseolamina comercial e a farinha de feijão como perspectiva ao tratamento da obesidade e do Diabetes mellitus tipo 2. [Dissertação]. Lavras: Universidade Federal de Lavras, 2008.

QIAN, M. et al. Molecular basis of the effects of chloride ion on the acid-base catalyst in the mechanism of pancreatic $\alpha$-Amylase. Biochemistry, v.44. n.9, p. 3194-3201, 2005. Disponível em:<http://www.ncbi.nlm.nih.gov/pubmed/15736930>. Acessado em: set. 2013.

RUAS, J. Conjuntura de 09.09.2010 para o Feijão. Brasília: Conab. Disponível em: <http://www.agricultura.gov.br/arq_editor/file/camaras_setoriais/Feijao/15_reuniao/C onsumo.pdf>. Acessado em: out. 2013.

UDANI, J.; SINGH, B.B. Blocking carbohydrate absorption and weight loss: a clinical trial using a proprietary fractionated white bean extract. Altern Ther Health Med. v.13, p. 32-37, 2007.

UNDANI, J.; HARDY, M.; MADSEN, D.C. Blocking carbohydrate absorption and weight loss: a clinical trial using Phase $2 ®$ brand proprietary fractionated white bean extract. Rev Altern Med. v.1, p. 63-69, 2004.

VIEIRA, C. O feijoeiro comum [tese livre docência]. Universidade Federal de Viçosa: Imprensa Universitária, 1967.

WU, W. True protein digestibility and digestibility-corrected amino acid score of red kidney beans (Phaseolus vulgaris L.). Journal of agricultural and Food Chemistry, Washington, v.43, n.5, p. 1295-1298, 1995. 